\title{
HYPERSURFACES OF PRESCRIBED CURVATURE MEASURE
}

\author{
PENGFEI GUAN, JUNFANG LI, AND YANYAN LI
}

\begin{abstract}
We consider the corresponding Christoffel-Minkowski problem for curvature measures. The existence of star-shaped $(n-k)$-convex bodies with prescribed $k$-th curvature measures $(k>0)$ has been a longstanding problem. This is settled in this paper through the establishment of a crucial $C^{2}$ a priori estimate for the corresponding curvature equation on $\mathbb{S}^{n}$.
\end{abstract}

\section{INTRODUCTION}

This paper concerns the general prescribing curvature measures problem. Curvature measures and area measures are two main subjects in convex geometry. They are the local versions of quermassintegrals in the Brunn-Minkowski theory. The Minkowski problem is a problem of prescribing a given $n$-th area measure. The general Christoffel-Minkowski problem is a problem of prescribing a given $k$-th area measure. There is a vast literature devoted to the study of this type of problems, and we refer to 1, 17, 15, 3, 6, 8, 14, 19, 10, 12 and references therein. We consider the corresponding Christoffel-Minkowski problem for the curvature measures, that is, the problem of prescribing curvature measures.

We first recall the definition of curvature measures and area measures, more detailed study can be found in literatures in convex geometry, e.g. Chapter 4 in 20. Classically, curvature and area measures were introduced for convex bodies (nonempty, compact, convex subsets of $\mathbb{R}^{n+1}$ ). Suppose $K$ is a convex body in $\mathbb{R}^{n+1}$. There are two notions of local parallel sets: given any Borel set $\beta \in \mathfrak{B}\left(\mathbb{R}^{n+1}\right)$, consider

$$
A_{\rho}(K, \beta):=\left\{x \in \mathbb{R}^{n+1} \mid 0<d(K, x) \leq \rho \text { and } p(K, x) \in \beta\right\}
$$

which is the set of all points $x \in \mathbb{R}^{n+1}$ for which the distance $d(K, x) \leq \rho$ and for which the nearest point $p(K, x)$ belongs to $\beta$. Alternatively, one may prescribe a Borel set $\beta \subset \mathbb{S}^{n}$ of unit vectors and then consider

$$
B_{\rho}(K, \omega):=\left\{x \in \mathbb{R}^{n+1} \mid 0<d(K, x) \leq \rho \text { and } u(K, x) \in \omega\right\}
$$

which is the set of all $x \in \mathbb{R}^{n+1}$ for which $d(K, x) \leq \rho$ and for which the unit vector $u(K, x)$ pointing from $p(K, x)$ to $x$ belongs to $\beta$.

By the theory of convex geometry (e.g., page 203 in [20]), the measures of the above local parallel sets are polynomials in the parameter $\rho$. More precisely,

1991 Mathematics Subject Classification. 53C23, 35J60, 53C42.

Key words and phrases. curvature measures, the Christoffel-Minkowski problem, $k$-convex starshaped domains, fully nonlinear elliptic equations.

Research of the first author was supported in part by NSERC Discovery Grant. Research of the second author was supported in part by NSF DMS-1007223. Research of the third author was supported in part by NSF DMS-0701545. 


$$
\begin{aligned}
\mathcal{H}^{n+1}\left(A_{\rho}(K, \beta)\right) & =\frac{1}{n+1} \sum_{m=0}^{n} \rho^{n+1-m}\left(\begin{array}{c}
n+1 \\
m
\end{array}\right) \mathcal{C}_{m}(K, \beta), \\
\mathcal{H}^{n+1}\left(B_{\rho}(K, \omega)\right) & =\frac{1}{n+1} \sum_{m=0}^{n} \rho^{n+1-m}\left(\begin{array}{c}
n+1 \\
m
\end{array}\right) S_{m}(K, \omega),
\end{aligned}
$$

for $\beta \in \mathfrak{B}\left(\mathbb{R}^{n+1}\right), \omega \in \mathfrak{B}\left(\mathbb{S}^{n}\right)$, and $\rho>0$. The coefficients in the polynomials (1.1) are defined as generalized curvature measures. Moreover, the measure $\mathcal{C}_{0}(K, \cdot), \cdots, \mathcal{C}_{n}(K, \cdot)$ are called curvature measures of the convex body $K$, and $S_{0}(K, \cdot), \cdots, S_{n}(K, \cdot)$ are called area measures of $K$.

It is possible to study curvature measures for more general sets. For example, Federer [7] introduced the curvature measure for sets of positive reach. Sets of positive reach are generalization of convex sets and smooth submanifolds, for which local parallel sets can be defined in such a way that their measure has a polynomial expansion, yielding a Steiner formula. There has been extensive study on the sets of positive reach and its further generalizations, see the literature in the notes and references of the book 20. The basic techniques rely on the geometric measure theory.

The problem of prescribing area measures is called the Christoffel-Minkowski problem. This problem has been extensively studied, we refer to 12 for an updated account. Similar to area measures, the problem of prescribing curvature measures has been discussed in the literature ( e.g. see note 8 on page 396 of [20]). The problem of prescribing 0-th curvature measure is the Alexandrov problem which is the counterpart of the Minkowski problem. This problem amounts to solving a Monge-Ampére type equation on $\mathbb{S}^{n}$. The existence and uniqueness of solutions were obtained by A.D. Alexandrov [2]. For $n=2$ the regularity of solutions of the Alexandrov problem in the elliptic case was proved by Pogorelov [18] and for higher dimensional cases, it was solved by Oliker [16. The general regularity results (degenerate case) of the problem were obtained in [10. The general problem of prescribing $k$-th curvature measure for $k \geq 0$ is an interesting counterpart of the Christoffel-Minkowski problem. The existence theorem for $k$-th curvature measures $(k>0)$ has been open up to now. The main objective of this paper is to settle this problem.

Let us start from an equivalent definition of the generalized curvature measures for convex bodies with smooth boundaries. Suppose that there is a bounded convex domain $\Omega \subset \mathbb{R}^{n+1}$ with $C^{2}$ boundary $M$. Let $\kappa=\left(\kappa_{1}, \cdots, \kappa_{n}\right)$ be the principal curvatures of $M$ at point $x, r=\left(r_{1}, \cdots, r_{n}\right)$ be the principal curvature radii, and $\sigma_{k}$ be the $k$-th elementary symmetric function. Then the $m$-th curvature measure and area measure of $\Omega$ have the following equivalent form

$$
\begin{aligned}
\mathcal{C}_{m}(\Omega, \beta) & :=\left(\begin{array}{c}
n \\
n-m
\end{array}\right)^{-1} \int_{\beta \cap M} \sigma_{n-m}(\kappa) d \mu_{g} \\
\mathcal{S}_{m}(\Omega, \omega) & :=\left(\begin{array}{c}
n \\
m
\end{array}\right)^{-1} \int_{\omega} \sigma_{m}(r) d \mathbb{S}^{n},
\end{aligned}
$$

for $\beta \in \mathbb{R}^{n+1}$ and $\omega \in \mathbb{S}^{n}$, where $d \mu_{g}$ is the volume element with respect to the induced metric $g$ of $M$ in $\mathbb{R}^{n+1}$, and $d \mathbb{S}^{n}$ is the volume element of the standard spherical metric. 
Note that the classical Christoffel-Minkowski problem for area measures is always confined to convex bodies, as the area measures are defined through Gauss map on $\mathbb{S}^{n}$. For the curvature measure $\mathcal{C}_{m}$ defined in (1.2), $\Omega$ is not necessarily convex if $m>0$. In the work of Alexandrov [2, the curvature measures is prescribed on $\mathbb{S}^{n}$ via radial map. With the radial parameterization of $\Omega$, the natural class is the star-shaped domains. In the rest of this paper, we treat the prescribing curvature measure problem for bounded star-shaped domains, including the convex bodies as a special case. In short, we prove the existence theorems of prescribing general $k$-th curvature measure problem with $k>0$ on bounded $C^{2}$ star-shaped domains.

Let $\Omega \subset \mathbb{R}^{n+1}$ be a bounded star-shaped domain with respect to the origin. Assume the boundary $M$ of $\Omega$ is $C^{2}$. Since $M$ is star-shaped, it can be viewed as a radial graph of $\mathbb{S}^{n}$, i.e.

$$
\begin{aligned}
R_{M}: \mathbb{S}^{n} & \longrightarrow M \\
z & \longmapsto \rho(z) z
\end{aligned}
$$

Following [2], up to a normalization constant, for each star-shaped domain $\Omega$ with $M=\partial \Omega$, the $m$-th curvature measure on each Borel set $\beta$ in $\mathbb{S}^{n}$ can be defined as

$$
\mathcal{C}_{m}(M, \beta):=\int_{R_{M}(\beta)} \sigma_{n-m}(\kappa) d \mu_{g} .
$$

In order that the curvature measure is a regular measure on $\mathbb{S}^{n}$, some geometric conditions on $M$ are necessary. Recall that the Garding's cone is defined as

$$
\Gamma_{k}=\left\{\lambda \in \mathbb{R}^{n} \mid \sigma_{i}(\lambda)>0, \forall i \leq k \cdot\right\} .
$$

A domain $\Omega \subset \mathbb{R}^{n+1}$ is called $k$-convex if its principal curvature vector $\kappa(x)=$ $\left(\kappa_{1}, \cdots, \kappa_{n}\right) \in \Gamma_{k}$ at every point $x \in \partial \Omega$. As the density of the $k$-th curvature measure is nonnegative, $M$ is $(n-k)$-convex. The corresponding Christoffel-Minkowski problem for curvature measures can be formulated as follows.

Q: For each $1 \leq k \leq n$ and each given positive function $f \in C^{2}\left(\mathbb{S}^{n}\right)$, find a closed hypersurface $M$ as a radial graph over $\mathbb{S}^{n}$, such that $\mathcal{C}_{n-k}(M, \beta)=\int_{\beta} f d \mu$ for every Borel set $\beta$ in $\mathbb{S}^{n}$, where $d \mu$ is the standard volume element on $\mathbb{S}^{n}$.

As the case $k=n$ is the Alexandrov problem which was completely settled, we are mainly interested in the cases $k<n$. The study of the problem $\mathbf{Q}$ was initiated by the first and third authors in [9]. Recently, with certain assumptions on $f$, Guan-Lin-Ma obtained the existence and regularity of convex solutions in [11. The curvature measure problem is equivalent to solving a fully nonlinear partial differential equation on $\mathbb{S}^{n}$, 9, 11]. For the $C^{2}$ graph $M$ on $\mathbb{S}^{n}$, denote the induced metric to be $g$ and the density function is $\sqrt{\operatorname{det} g}$. Then

$$
\mathcal{C}_{n-k}(M, \beta)=\int_{R_{M}(\beta)} \sigma_{k} d \mu_{g}=\int_{\beta} \sigma_{k} \sqrt{\operatorname{det} g} d \mathbb{S}^{n} .
$$

The above density function can be computed as (see computations in the next section (2.5))

$$
\sqrt{\operatorname{det} g}=\rho^{n-1} \sqrt{\rho+|\nabla \rho|^{2}}
$$

where the covariant derivative $\nabla$ is with respect to the standard spherical metric. Therefore, we can reduce the prescribing $(n-k)$-th curvature measure problem to the following curvature equation on $\mathbb{S}^{n}$ :

$$
\sigma_{k}\left(\kappa_{1}, \cdots, \kappa_{n}\right)=\frac{f}{\rho^{n-1} \sqrt{\rho+|\nabla \rho|^{2}}},
$$


where $f>0$ is the given function on $\mathbb{S}^{n}$. A solution of (1.4) is called admissible if $\kappa(X) \in \Gamma_{k}$ at each point $X \in M$. We note that any positive $C^{2}$ function $\rho$ on $\mathbb{S}^{n}$ satisfying equation (1.4) is automatically an admissible solution since $f>0$ and principal curvatures at a maximum point of $\rho$ are non-negative. Equation (1.4) is a special type of fully nonlinear partial differential equations studied in the pioneer work by Caffarelli-Nirenberg-Spruck [4, 5.

$C^{0}$ and $C^{1}$ estimates for admissible solutions along with the uniqueness of admissible solutions for equation (1.4) were proved in the unpublished notes [9] by the first and third authors. For the existence of admissible solutions, the case $k=1$ follows from theories of quasi-linear elliptic equations [9]. When $k=n$, admissible solutions are convex and they were dealt with in [18, 16, 10. The existence of admissible solutions for the remaining cases, i.e., $1<k<n$, has been open due to the lack of $C^{2}$ a priori estimate for admissible solutions of (1.4).

We now state the main results. Our first theorem establishes the existence of admissible solutions for $1 \leq k<n$.

Theorem 1.1. Let $n \geq 2$ and $1 \leq k \leq n-1$. Suppose $f \in C^{2}\left(\mathbb{S}^{n}\right)$ and $f>0$. Then there exists a unique $k$-convex star-shaped hypersurface $M \in C^{3, \alpha}, \forall \alpha \in(0,1)$ such that it satisfies (1.4). Moreover, there is a constant $C$ depending only on $k, n,\|f\|_{C^{1,1}},\|1 / f\|_{C^{0}}$, and $\alpha$ such that,

$$
\|\rho\|_{C^{3, \alpha}} \leq C \text {. }
$$

It is of interest to find convex solutions to (1.4), namely, to prove existence results for the prescribing curvature measure problem for convex bodies. From the uniqueness of admissible solutions, solutions to equation (1.4) are not convex in general, except for the case $k=n$. For $k<n$, there has been some progress recently. In [11], Guan-Lin-Ma proved $C^{2}$ estimates and existence theorems for convex solutions of equation (1.4) for $1 \leq k<n$ under suitable convexity conditions of $f$. More specifically, they proved

Theorem A. (Curvature measure problem under strict convexity condition [1]) Suppose $f \in C^{2}\left(\mathbb{S}^{n}\right), f>0, n \geq 2,1 \leq k \leq n-1$. If $f$ satisfies that

$$
|X|^{\frac{n+1}{k}} f\left(\frac{X}{|X|}\right)^{-\frac{1}{k}} \text { is a strictly convex function in } \mathbb{R}^{n+1} \backslash\{0\},
$$

then there exists a unique strictly convex hypersurface $M \in C^{3, \alpha}, \alpha \in(0,1)$ such that it satisfies (1.4).

Moreover, if $k=1$, or 2, i.e., the cases of mean curvature measure and scalar curvature measure, condition (1.6) can be weakened as below.

Theorem B. (Mean curvature measure and scalar curvature measure under convexity condition [11) Suppose $k=1$, or 2 , and $k<n$. Suppose $f \in C^{2}\left(\mathbb{S}^{n}\right)$ is a positive function. If $f$ satisfies that

$$
|X|^{\frac{n+1}{k}} f\left(\frac{X}{|X|}\right)^{-\frac{1}{k}} \text { is a convex function in } \mathbb{R}^{n+1} \backslash\{0\},
$$

then there exists a unique strictly convex hypersurface $M \in C^{3, \alpha}, \alpha \in(0,1)$ such that it satisfies (1.4).

With the a priori estimates for admissible solutions of equation (1.4) in Theorem 1.1. we obtain the existence of convex bodies under weaker condition (1.7) for all $1 \leq k<n$. 
Theorem 1.2. Suppose $1 \leq k<n$ and $f \in C^{2}\left(\mathbb{S}^{n}\right)$ is a positive function. If $f$ satisfies

$$
|X|^{\frac{n+1}{k}} f\left(\frac{X}{|X|}\right)^{-\frac{1}{k}} \text { is a convex function in } \mathbb{R}^{n+1} \backslash\{0\},
$$

then there exists a unique strictly convex hypersurface $M \in C^{3, \alpha}, \alpha \in(0,1)$ such that it satisfies (1.4).

The rest of this paper is organized as follows. In Section 2, we fix notations and list necessary formulas we need. In Section 3, we establish the crucial $C^{2}$ estimate and prove Theorem 1.1 and Theorem 1.2

\section{Preliminaries}

We first recall the relevant geometric quantities for a smooth closed hypersurface in $\mathbb{R}^{n+1}$ we may need. Throughout the paper, repeated indices denote summation and we assume the origin is inside the domain enclosed by $M$.

Let $M^{n}$ be an immersed hypersurface in $\mathbb{R}^{n+1}$. For $X \in M \subset \mathbb{R}^{n+1}$, choose local normal coordinates in $\mathbb{R}^{n+1}$, such that $\left\{\frac{\partial}{\partial x_{1}}, \cdots, \frac{\partial}{\partial x_{n}}\right\}$ are tangent to $M$ and $\partial_{n+1}$ is the unit outer normal of the hypersurface. We sometimes denote $\partial_{i}:=\frac{\partial}{\partial x_{i}}$ and also use $\nu$ to denote the unit outer normal $\partial_{n+1}$. We use lower indices to denote covariant derivatives with respect to the induced metric.

For the immersion $X$, the second fundamental form is the symmetric $(2,0)$-tensor given by the matrix $\left\{h_{i j}\right\}$,

$$
h_{i j}=\left\langle\partial_{i} X, \partial_{j} \nu\right\rangle .
$$

Recall the following identities:

$$
\begin{aligned}
X_{i j} & =-h_{i j} \nu \quad \text { (Gauss formula) } \\
(\nu)_{i} & =h_{i j} \partial_{j} \quad \text { (Weigarten equation) } \\
h_{i j k} & =h_{i k j} \quad(\text { Codazzi formula) } \\
R_{i j k l} & =h_{i k} h_{j l}-h_{i l} h_{j k} \quad \text { (Gauss equation) }
\end{aligned}
$$

where $R_{i j k l}$ is the $(4,0)$-Riemannian curvature tensor. We also have

$$
\begin{aligned}
h_{i j k l} & =h_{i j l k}+h_{m j} R_{i m l k}+h_{i m} R_{j m l k} \\
& =h_{k l i j}+\left(h_{m j} h_{i l}-h_{m l} h_{i j}\right) h_{m k}+\left(h_{m j} h_{k l}-h_{m l} h_{k j}\right) h_{m i} .
\end{aligned}
$$

In certain cases of this article, we need to carry out calculations in a neighborhood of the standard sphere $\mathbb{S}^{n}$. Since $M$ is star-shaped with respect to the origin, the position vector $X$ can be written as $X(x)=\rho(x) x, x \in \mathbb{S}^{n}$ for some smooth function $\rho$ on $\mathbb{S}^{n}$. In this case, suppose $\left\{\partial_{1}, \cdots, \partial_{n}\right\}$ is some local normal coordinates on $\mathbb{S}^{n}$ and $\nabla$ is the covariant differentiation with respect to the standard metric on $\mathbb{S}^{n}$. Then the induced metric $g_{i j}$ of $M$ is given by

$$
g_{i j}=\rho^{2} \delta_{i j}+\rho_{i} \rho_{j},
$$

and the area density function

$$
\sqrt{\operatorname{det} g}=\rho^{n-1} \sqrt{\rho^{2}+|\nabla \rho|^{2}} .
$$

The second fundamental form of $M$ can be calculated as

$$
h_{i j}=\left(\rho^{2}+|\nabla \rho|^{2}\right)^{\frac{1}{2}}\left(\rho^{2} \delta_{i j}+2 \rho_{i} \rho_{j}-\rho \rho_{i j}\right)
$$


and the unit outer normal vector is

$$
\nu=\frac{\rho x-\nabla \rho}{\sqrt{\rho^{2}+|\nabla \rho|^{2}}}
$$

The support function of a hypersurface is defined as $u(X)=\langle X, \nu\rangle$. We can also compute it as

$$
u=\rho^{2}\left(\rho^{2}+|\nabla \rho|^{2}\right)^{-\frac{1}{2}} .
$$

The principal curvatures $\left(\kappa_{1}, \cdots, \kappa_{n}\right)$ are the eigenvalues of the second fundamental form with respect to the metric which satisfy

$$
\operatorname{det}\left(h_{i j}-\kappa g_{i j}\right)=0 .
$$

The curvature equation (1.4) on $\mathbb{S}^{n}$ can also be equivalently expressed as an equation of the position vector $X$. Using (2.7), we have

$$
\sigma_{k}\left(\kappa_{1}, \cdots, \kappa_{n}\right)(X)=\frac{u(X)}{|X|^{n+1}} f\left(\frac{X}{|X|}\right), \quad \forall X \in M .
$$

The uniqueness results, $C^{0}, C^{1}$ estimates for solutions of equation (1.4) were proved in [9] (see also [11]). For completeness, we state the results here. The first lemma concerns the uniqueness of solutions.

Lemma 2.1. Let $1 \leq k<n$. Suppose $\rho_{1}, \rho_{2}$ are two solutions of equation (1.4) and $\lambda\left(\rho_{i}\right) \in \Gamma_{k}$, for $i=1,2$. Then $\rho_{1} \equiv \rho_{2}$.

The following lemma will be useful later in this paper. Set $F(\lambda)=\sigma_{k}(\lambda)^{\frac{1}{k}} . F(\lambda)$ is homogeneous of degree one. Equation (1.4) can be written as

$$
F(\lambda) \equiv F\left(\lambda_{1}, \cdots, \lambda_{n}\right)=f^{\frac{1}{k}} \rho^{\frac{(1-n)}{k}}\left(\rho^{2}+|\nabla \rho|^{2}\right)^{-1 /(2 k)} \equiv K(x, \rho, \nabla \rho) .
$$

Lemma 2.2. Let $L$ denote the linearized operator of $F(\lambda)-K(x, \rho, \nabla \rho)$ at a solution $\rho$ of (1.4). If $\omega$ satisfies $L \omega=0$ on $\mathbb{S}^{n}$, then $\omega \equiv 0$ on $\mathbb{S}^{n}$.

Next is the $C^{0}, C^{1}$ estimates.

Lemma 2.3. If $M$ satisfies (2.9), then

$$
\left(\frac{\min _{\mathbb{S}^{n}} f}{C_{n}^{k}}\right)^{\frac{1}{n-k}} \leq \min _{\mathbb{S}^{n}}|X| \leq \max _{\mathbb{S}^{n}}|X| \leq\left(\frac{\max _{\mathbb{S}^{n}} f}{C_{n}^{k}}\right)^{\frac{1}{n-k}} .
$$

Moreover, there exits a constant $C$ depending only on $n, k, \min _{\mathbb{S}^{n}} f,|f|_{C^{1}}$ such that

$$
\max _{\mathbb{S}^{n}}|\nabla \rho| \leq C .
$$

\section{THE $C^{2}$ A PRIORI ESTIMATES AND THE PROOF OF MAIN RESUlts}

The major step of this paper is to establish the a priori $C^{2}$-estimates. Equation (1.4) is similar to the prescribing curvature equation treated in [5. There, $C^{2}$ estimates were proved for a general type of curvature equations using the concavity of $\sigma^{\frac{1}{k}}$. Equation (1.4) differs from the equations treated in [5], as the right hand side of (1.4) depends on $\nabla \rho$ too. We do not see how to apply the arguments in [5] to deal with equation (1.4). Under certain structural convexity conditions, $C^{2}$-estimates were obtained for convex solutions of (1.4) in 11]. For admissible solutions, this has been a missing piece for a while. In this section, we prove a key Lemma 3.2 to 
overcome the main difficulty in the proof of $C^{2}$ estimate for admissible solutions. We believe that Lemma 3.2 will be useful to treat other curvature problems as well.

Proposition 3.1. For $1<k<n$, let $F \equiv \sigma_{k}=\phi u$ and denote $H \equiv \sigma_{1}$, then at a maximum point of $\frac{H}{u}$,

$$
\begin{aligned}
F^{i j}\left(\frac{H}{u}\right)_{i j}= & \frac{1}{u}\left[\phi_{s s} u+2 \phi_{s} u_{s}\right]-\left(\frac{H}{u}\right) \phi_{l}\left\langle X, X_{l}\right\rangle-(k-1)\left(\frac{H}{u}\right) \phi \\
& +(k-1) \phi|A|^{2}-\frac{1}{u} F^{i j ; m l} h_{i j ; s} h_{m l ; s},
\end{aligned}
$$

where $A$ denotes the second fundamental form.

Proof. We will need the following formulas.

$$
\begin{aligned}
u_{s} & =h_{s l}\left\langle X, X_{l}\right\rangle \\
u_{i j} & =h_{i j ; l}\left\langle X, X_{l}\right\rangle+h_{i j}-\left(h^{2}\right)_{i j} u \\
h_{i j ; k l} & =h_{k l ; i j}+\left(h_{l k} h_{i m}-h_{l m} h_{i k}\right) h_{m j}+\left(h_{l j} h_{i m}-h_{l m} h_{i j}\right) h_{m k} \\
F^{i j} h_{i j ; s t} & =F_{s t}-F^{i j ; m l} h_{m l ; s} h_{i j ; t} .
\end{aligned}
$$

Assume that $\frac{H}{u}$ achieves the maximal value at some point $P \in M^{n}$ which implies $\left(\frac{H}{u}\right)_{i}(P)=0$. In the rest of the proof, all calculations will be performed at the maximum point $P$. First, since $\left(\frac{H}{u}\right)_{i}=0$, we have

$$
\begin{aligned}
F^{i j}\left(\frac{H}{u}\right)_{i j} & =F^{i j}\left[\frac{H_{i j}}{u}-\frac{u_{j}}{u}\left(\frac{H}{u}\right)_{i}-\frac{u_{i}}{u}\left(\frac{H}{u}\right)_{j}-\left(\frac{H}{u}\right) \frac{u_{i j}}{u}\right] \\
& =\frac{1}{u} F^{i j} H_{i j}-\frac{1}{u}\left(\frac{H}{u}\right) F^{i j} u_{i j} .
\end{aligned}
$$

On the other hand, applying formulas in (3.2), we have

$$
\begin{aligned}
\frac{1}{u} F^{i j} H_{i j}= & \frac{1}{u} F^{i j} h_{s s ; i j} \\
= & \frac{1}{u} F^{i j}\left[h_{i j ; s s}+\left(h_{i j} h_{s m}-h_{j m} h_{s i}\right) h_{m s}+\left(h_{j s} h_{s m}-h_{j m} h_{s s}\right) h_{m i}\right] \\
= & \frac{1}{u} F^{i j} h_{i j ; s s}+k \phi|A|^{2}-\frac{1}{u} F^{i j}\left(h^{2}\right)_{i j} H \\
= & \frac{1}{u} F_{s s}-\frac{1}{u} F^{i j ; m l} h_{i j ; s} h_{m l ; s}+k \phi|A|^{2}-\left(\frac{H}{u}\right) F^{i j}\left(h^{2}\right)_{i j} \\
= & \frac{1}{u}\left[\phi_{s s} u+2 \phi_{s} u_{s}+\phi u_{s s}\right]-\frac{1}{u} F^{i j ; m l} h_{i j ; s} h_{m l ; s}+k \phi|A|^{2} \\
= & \left.\frac{1}{u}\left[\frac{H}{u}\right) F^{i j}\left(h^{2}\right)_{i j} u+2 \phi_{s} u_{s}\right]+\frac{\phi}{u}\left[H_{l}\left\langle X, X_{l}\right\rangle+H-|A|^{2} u\right] \\
& -\frac{1}{u} F^{i j ; m l} h_{i j ; s} h_{m l ; s}+k \phi|A|^{2}-\left(\frac{H}{u}\right) F^{i j}\left(h^{2}\right)_{i j} \\
= & \frac{1}{u}\left[\phi_{s s} u+2 \phi_{s} u_{s}\right]+\frac{\phi}{u} H_{l}\left\langle X, X_{l}\right\rangle+\left(\frac{H}{u}\right) \phi \\
& -\frac{1}{u} F^{i j ; m l} h_{i j ; s} h_{m l ; s}+(k-1) \phi|A|^{2}-\left(\frac{H}{u}\right) F^{i j}\left(h^{2}\right)_{i j} .
\end{aligned}
$$

We also compute the following

$$
\begin{aligned}
-\frac{1}{u}\left(\frac{H}{u}\right) F^{i j} u_{i j} & =-\frac{1}{u}\left(\frac{H}{u}\right) F^{i j}\left[h_{i j ; l}\left\langle X, X_{l}\right\rangle+h_{i j}-\left(h^{2}\right)_{i j} u\right] \\
& =-\frac{1}{u}\left(\frac{H}{u}\right) F_{l}\left\langle X, X_{l}\right\rangle-k \phi\left(\frac{H}{u}\right)+\left(\frac{H}{u}\right) F^{i j}\left(h^{2}\right)_{i j} \\
& =-\frac{\phi}{u}\left(\frac{H}{u}\right) u_{l}\left\langle X, X_{l}\right\rangle-\left(\frac{H}{u}\right) \phi_{l}\left\langle X, X_{l}\right\rangle-k \phi\left(\frac{H}{u}\right)+\left(\frac{H}{u}\right) F^{i j}\left(h^{2}\right)_{i j},
\end{aligned}
$$

where $\left(h^{2}\right)_{i j}=h_{i k} h_{k j}$.

Adding up (3.4) and (3.5), and using the critical point condition, we obtain 


$$
\begin{aligned}
F^{i j}\left(\frac{H}{u}\right)_{i j}= & \frac{1}{u}\left[\phi_{s s} u+2 \phi_{s} u_{s}\right]+\phi\left(\frac{H}{u}\right)_{l}\left\langle X, X_{l}\right\rangle-\left(\frac{H}{u}\right) \phi_{l}\left\langle X, X_{l}\right\rangle \\
& -(k-1)\left(\frac{H}{u}\right) \phi-\frac{1}{u} F^{i j ; m l} h_{i j ; s} h_{m l ; s}+(k-1) \phi|A|^{2} \\
= & \frac{1}{u}\left[\phi_{s s} u+2 \phi_{s} u_{s}\right]-\left(\frac{H}{u}\right) \phi_{l}\left\langle X, X_{l}\right\rangle-(k-1)\left(\frac{H}{u}\right) \phi \\
& -\frac{1}{u} F^{i j ; m l} h_{i j ; s} h_{m l ; s}+(k-1) \phi|A|^{2},
\end{aligned}
$$

which finishes the proof.

The following lemma is the key for $C^{2}$-estimates.

Lemma 3.2. Let $\alpha=\frac{1}{k-1}$, if $\left(h_{i j}\right) \in \Gamma_{k}$, then

$$
\left(\sigma_{k}\right)^{i j, l m} h_{i j ; s} h_{i j ; s} \leq-\sigma_{k}\left[\frac{\left(\sigma_{k}\right)_{s}}{\sigma_{k}}-\frac{\left(\sigma_{1}\right)_{s}}{\sigma_{1}}\right]\left[(\alpha-1) \frac{\left(\sigma_{k}\right)_{s}}{\sigma_{k}}-(\alpha+1) \frac{\left(\sigma_{1}\right)_{s}}{\sigma_{1}}\right] .
$$

Proof. By the concavity of $\left(\frac{\sigma_{k}}{\sigma_{1}}\right)^{\frac{1}{k-1}}\left(h_{i j}\right)$, we have

$$
0 \geq \frac{\partial^{2}}{\partial h_{i j} \partial h_{l m}}\left(\left(\frac{\sigma_{k}}{\sigma_{1}}\right)^{\frac{1}{k-1}}\right) h_{i j ; s} h_{l m ; s} .
$$

To simplify notations, we denote $\alpha=\frac{1}{k-1}$. Direct computations yield,

$$
\begin{aligned}
0 \geq & \frac{\partial^{2}}{\partial h_{i j} \partial h_{l m}}\left(\frac{\sigma_{k}}{\sigma_{1}}\right)^{\alpha} \cdot h_{i j ; s} h_{l m ; s} \\
= & \alpha\left(\frac{\sigma_{k}}{\sigma_{1}}\right)^{\alpha}\left[\frac{\left(\sigma_{k}\right)^{i j, l m}}{\sigma_{k}}+\frac{(\alpha-1)\left(\sigma_{k}\right)^{i j}\left(\sigma_{k}\right)^{l m}}{\sigma_{k}^{2}}\right. \\
& \left.-\frac{2 \alpha\left(\sigma_{k}\right)^{i j}\left(\sigma_{1}\right)^{l m}}{\sigma_{k} \sigma_{1}}+\frac{(\alpha+1)\left(\sigma_{1}\right)^{i j}\left(\sigma_{1}\right)^{l m}}{\sigma_{1}^{2}}\right] h_{i j ; s} h_{l m ; s}
\end{aligned}
$$

Equivalently,

$$
\begin{aligned}
\frac{\left(\sigma_{k}\right)^{i j, l m} h_{i j ; s} h_{l m ; s} \leq}{\sigma_{k}} \leq & -\left[\frac{(\alpha-1)\left(\sigma_{k}\right)^{i j}\left(\sigma_{k}\right)^{l m}}{\sigma_{k}^{2}}-\frac{2 \alpha\left(\sigma_{k}\right)^{i j}\left(\sigma_{1}\right)^{l m}}{\sigma_{k} \sigma_{1}}\right. \\
& \left.+\frac{(\alpha+1)\left(\sigma_{1}\right)^{i j}\left(\sigma_{1}\right)^{l m}}{\sigma_{1}^{2}}\right] h_{i j ; s} h_{l m ; s} \\
\leq & -\left[\frac{\left(\sigma_{k}\right)_{s}}{\sigma_{k}}-\frac{\left(\sigma_{1}\right)_{s}}{\sigma_{1}}\right]\left[(\alpha-1) \frac{\left(\sigma_{k}\right)_{s}}{\sigma_{k}}-(\alpha+1) \frac{\left(\sigma_{1}\right)_{s}}{\sigma_{1}}\right]
\end{aligned}
$$

Note in Lemma 3.2. property that $\left(\frac{F}{\sigma_{1}}\right)^{\alpha}$ is concave for some $\alpha>0$. The following is a corollary of Lemma 3.2

Corollary 3.3. If $\frac{\left(\sigma_{1}\right)_{s}}{\sigma_{1}}=\frac{\left(\sigma_{k}\right)_{s}}{\sigma_{k}}-r$ for some $r$,

$$
\left(\sigma_{k}\right)^{i j, l m} h_{i j ; s} h_{i j ; s} \leq \max \left\{2 r\left(\sigma_{k}\right)_{s}-\frac{k}{k-1} r^{2} \sigma_{k}, 0\right\} .
$$

Combining Proposition 3.1 and Corollary 3.3 , we obtain the following $C^{2}$ estimates. 
Lemma 3.4. If $M$ satisfies equation (2.9) and $2 \leq k \leq n$, then there exists a constant $C$ depending only on $n, k, \min _{S^{n}} f,|f|_{C^{1}}$, and $|f|_{C^{2}}$, such that

$$
\max _{M} \sigma_{1} \leq C, \quad \nabla^{2} \rho \leq C .
$$

Proof. We have already obtained the $C^{0}$ and $C^{1}$ estimates for $\rho$. Therefore, to bound $\nabla^{2} \rho$, one only need to obtain an estimate for $\sigma_{1}\left(h_{j}^{i}\right)=g^{i j} h_{i j}$ in view of $\sigma_{1}^{2}=|A|^{2}+2 \sigma_{2}$ and $\sigma_{2}>0$, where $h_{j}^{i}=g^{i k} h_{k j}$ is the Weingarten tensor.

If we denote $H \doteq \sigma_{1}$, then at a point $P$ where $\frac{H}{u}$ achieves its maximum, we have shown in (3.1)

$$
\begin{aligned}
0 \geq & F^{i j}\left(\frac{H}{u}\right)_{i j} \\
= & \frac{1}{u}\left[\phi_{s s} u+2 \phi_{s} u_{s}\right]-\left(\frac{H}{u}\right) \phi_{l}\left\langle X, X_{l}\right\rangle-(k-1)\left(\frac{H}{u}\right) \phi \\
& -\frac{1}{u} F^{i j ; m l} h_{i j ; s} h_{m l ; s}+(k-1) \phi|A|^{2} .
\end{aligned}
$$

Recall that $\phi(X) \in C^{2}(M)$ is defined as $\phi(X)=|X|^{-(n+1)} f\left(\frac{X}{|X|}\right)$ and $C^{0}, C^{1}$ estimates of $\rho=|X|$ are already known. We have the following estimates.

$$
\begin{aligned}
\left|\phi_{i}\right|(P) & \leq C\left(n, k, \min _{S^{n}} f,|f|_{C^{1}}\right) \\
\left|\phi_{i i}\right|(P) & \leq C\left(n, k, \min _{S^{n}} f,|f|_{C^{1}},|f|_{C^{2}}\right)(1+|A|(P))
\end{aligned}
$$

On the other hand, $\left|u_{i}\right|=\left|h_{j}^{i} \rho \rho_{j}\right| \leq c_{3}|A|$. Observe that by equation (2.9), $\frac{\sigma_{1}}{u}=\frac{\sigma_{1} \phi}{\sigma_{k}}$. At a maximum point of the test function $\frac{\sigma_{1}}{u}$, one has $\frac{\left(\sigma_{1}\right)_{s}}{\sigma_{1}}=\frac{\left(\sigma_{k}\right)_{s}}{\sigma_{k}}-\frac{\phi_{s}}{\phi}$. In Corollary 3.3 let $r=\frac{\phi_{s}}{\phi}(P)$, then

$$
\begin{aligned}
F^{i j ; m l} h_{i j ; s} h_{m l ; s} & \leq 2 r(u \phi)_{s}-\frac{k}{k-1} r^{2} u \phi \\
& \leq C_{1}\left(n, k, \min _{S^{n}} f,|f|_{C^{1}}\right)|A|+C_{2}\left(n, k, \min _{S^{n}} f,|f|_{C^{1}}\right) .
\end{aligned}
$$

With the above estimates, we simplify (3.13) to be

$$
|A|^{2}(P)+c_{4}|A|(P)+c_{5} \leq 0,
$$

where $c_{4}$ and $c_{5}$ are constants depending only on $n, k, \min _{S^{n}} \phi,|f|_{C^{1}}$, and $|f|_{C^{2}}$. Hence at $P,|A|(P) \leq C$. In turn $\sigma_{1}(X) \leq u(X) \frac{\sigma_{1}(P)}{u(P)} \leq C$ for any $X \in M$. This implies (3.12).

Remark 3.5. At this point, we would like to raise a question regarding global $C^{2}$ estimates for general curvature equations. To be precise, suppose $M \subset \mathbb{R}^{n+1}$ is a compact smooth hypersurface satisfying equation

$$
\sigma_{k}(\kappa(X))=f(X, \nu), \kappa(X) \in \Gamma_{k}, \quad \forall X \in M,
$$

where $f \in C^{2}\left(\mathbb{R}^{n+1} \times \mathbb{S}^{n}\right)$ is a general positive function. Suppose there is a priori $C^{1}$ bound of $M$, can one conclude a $C^{2}$ a priori bound of $M$ in terms of $C^{1}$ norm of $M, f, n, k$ ? When $k=1$, the answer is affirmative. It follows from theories of quasilinear elliptic equations, in particular that of the mean curvature type equation. When $k=n$, this is also true (e.g., Theorem 5.1 in [11]), it is parallel to a well known fact for Monge-Ampère equation.

A similar question can also be asked for Hessian equations $(1<k<n)$. Suppose that $u$ is an admissible solution of equation

$$
\sigma_{k}\left(\nabla^{2} u\right)=f(x, u, \nabla u), \forall x \in \Omega \subset \mathbb{R}^{n},
$$


where $f \in C^{2}\left(\Omega \times \mathbb{R} \times \mathbb{R}^{n}\right)$ is a general positive function, is it true that there is $C$ depending only on $n, k,\|f\|_{C^{1,1}},\|1 / f\|_{C^{0}},\|u\|_{C^{0,1}(\bar{\Omega})}$ such that

$$
\max _{x \in \Omega}\left|\nabla^{2} u(x)\right| \leq C\left(1+\max _{x \in \partial \Omega}\left|\nabla^{2} u(x)\right|\right) ?
$$

Now we are ready to prove the main theorems of this paper.

Proof of Theorem 1.1. For any positive function $f \in C^{2}\left(\mathbb{S}^{n}\right)$, for $0 \leq t \leq 1$ and $1<k<n-1$, set $f_{t}(x)=\left[1-t+t f^{-\frac{1}{k}}(x)\right]^{-k}$. We consider the following family of equations for $0 \leq t \leq 1$ :

$$
\sigma_{k}\left(\kappa_{1}, \cdots, \kappa_{n}\right)(x)=f_{t}(x) \rho^{1-n}\left(\rho^{2}+|\nabla \rho|^{2}\right)^{-1 / 2}, \quad \text { on } \mathbb{S}^{n},
$$

where $n \geq 2$. We want to find admissible solutions in the class of star-shaped hypersurfaces. Let $I=\{t \in[0,1]$ : such that (3.18) is solvable $\}$. $I$ is nonempty because $\rho=\left[C_{n}^{k}\right]^{-\frac{1}{n-2}}$ is a solution for $t=0$. By the a priori estimates of lemmas 2.3 and 3.4 the Evans-Krylov theorem and the Schauder theorem, $\rho \in C^{3, \alpha}\left(\mathbb{S}^{n}\right)$ and

$$
\|\rho\|_{C^{3, \alpha}\left(\mathbb{S}^{n}\right)} \leq C
$$

where $C$ depends only on only on $n, k, \min _{S^{n}} f, \max _{S^{n}} f,|f|_{C^{1}},|f|_{C^{2}}$ and $\alpha$. The a priori estimates guarantee that $I$ is closed. The openness comes from Lemma 2.2 and the implicit function theorem. This proves the existence part of the theorem. The uniqueness part of the theorem follows from Lemma 2.1. The proof of Theorem 1.1 is completed.

To prove Theorem 1.2, we need the following Constant Rank Theorem in [11].

Theorem 3.6. 11] Suppose $M$ is a convex hypersurface and satisfies equation (2.9) for $k<n$ with the second fundamental form $W=\left\{h_{i j}\right\}$ and $|X|^{\frac{n+1}{n}} f\left(\frac{X}{|X|}\right)$ is convex in $\mathbb{R}^{n+1} \backslash\{0\}$, then $W$ is positive definite.

Proof of Theorem 1.2, By the proof of Theorem 1.1, there exits a unique admissible solution to (2.9). We need to prove the strict convexity of solutions under the weak convexity condition (1.8) of $|X|^{\frac{n+1}{k}} f\left(\frac{X}{|X|}\right)^{-\frac{1}{k}}$. Using the same deformation path as in the proof of Theorem 1.1, we start from the sphere solution with $\rho=\left[C_{n}^{k}\right]^{\frac{1}{n-2}}$ and prove the theorem by contradiction. Suppose that the strict convexity is not true. By continuity, there must exist a first $t_{0} \in[0,1]$ such that the second fundamental form $W(x, t)>0$ for all $x \in M, t \in\left[0, t_{0}\right)$. Moreover, at $t=t_{0}, W\left(x, t_{0}\right) \geq 0$ for all $x \in M$ and there exists $x_{0} \in M$ such that $W\left(x_{0}, t_{0}\right)=0$. However, since the hypersurface $M\left(t_{0}\right)$ is convex, by Theorem 3.6, $M\left(x, t_{0}\right)$ must be strictly convex for any $x \in M\left(t_{0}\right)$ which contradicts $W\left(x_{0}, t_{0}\right)=0$. This proves that the solution hypersurface to (2.9) must be strictly convex.

The condition in Theorem 1.2 can be weakened further following the same compactness argument in the proof of Theorem 5 in [13].

Theorem 3.7. Suppose $1 \leq k<n$ and $f \in C^{2}\left(\mathbb{S}^{n}\right)$ is a positive function. There is $\delta>0$ depending only on $n, k,\|f\|_{C^{1,1}},\|1 / f\|_{C^{0}}$ such that, if $f$ satisfies

$$
|X|^{\frac{n+1}{k}} f\left(\frac{X}{|X|}\right)^{-\frac{1}{k}}+\delta|X|^{2} \text { is a convex function in } \mathbb{R}^{n+1} \backslash\{0\} \text {, }
$$


then there exists a unique strictly convex hypersurface $M \in C^{3, \alpha}, \alpha \in(0,1)$ such that it satisfies (1.4).

Proof. Suppose it is not true. In view of Theorem 1.1, there is a constant $C>0$ and a sequence of positive functions $f_{l}$ on $\mathbb{S}^{n}$ with

$$
\begin{aligned}
& \left\|f_{l}\right\|_{C^{1,1}} \leq C, \quad\left\|\frac{1}{f_{l}}\right\|_{C^{0}} \leq C, \\
& |X|^{\frac{n+1}{k}} f_{l}\left(\frac{X}{|X|}\right)^{-\frac{1}{k}}+\frac{1}{l}|X|^{2} \text { convex in } \mathbb{R}^{n+1} \backslash\{0\},
\end{aligned}
$$

and a sequence of admissible solutions $M_{l}=\left\{X=\rho_{l}\left(\frac{X}{|X|}\right) X\right\}$ with principal curvature vector $\kappa^{l}$ satisfying

$$
\sigma_{k}\left(\kappa_{1}^{l}, \cdots, \kappa_{n}^{l}\right)=\frac{f_{l}}{\rho_{l}^{n-1} \sqrt{\rho_{l}+\left|\nabla \rho_{l}\right|^{2}}},
$$

and a sequence of points $x_{l} \in \mathbb{S}^{n}$ such that

$$
\kappa_{j(l)}^{l}\left(x_{l}\right) \leq 0
$$

Again by Theorem 1.1, there is some $\tilde{C}$ depending on on $k, n,\left\|f_{l}\right\|,\left\|\frac{1}{f_{l}}\right\|_{C^{0}}, \alpha$ such that

$$
\|\rho\|_{C^{3, \alpha}} \leq \tilde{C}
$$

By the compactness, there exist subsequences which we still denote $x_{l}, f_{l}, \rho_{l}$ such that

$$
x_{l} \rightarrow x_{0} \in \mathbb{S}^{n}, \quad f_{l} \rightarrow f \in C^{1, \alpha}, \quad \rho_{l} \rightarrow \rho \in C^{3, \alpha}
$$

with $\rho$ satisfying equation (1.4). Clearly, $f \in C^{1,1}$ and it satisfies condition (1.8). By Theorem 1.2 $M$ is strictly convex. But from (3.20), $M$ is not strictly convex at $x_{0}$. This is a contradiction.

\section{REFERENCES}

[1] Alexandrov, A. D. Zur theorie der gemishchten volumina von knovexen korpern, 2, Mathematics of the USSR Sbornik 2 (1937), 1205-38.

[2] Alexandrov, A. D. Existence and uniqueness of a convex surface with a given integral curvature, Doklady Akademii Nauk Kasah SSSR 36 (1942), 131-34.

[3] Berg, Christian. Corps convexes et potentiels sphëriques, (French) Mat.-Fys. Medd. Danske Vid. Selsk. 371969 no. 6, 64 pp. (1969).

[4] Caffarelli, Luis, Nirenberg, Louis, and Spruck, Joel. The Dirichlet problem for nonlinear second order elliptic equations, III: Functions of the eigenvalues of the Hessian Acta Math. 155 (1985), 261-301.

[5] Caffarelli, Luis, Nirenberg, Louis, and Spruck, Joel. Nonlinear second order elliptic equations IV: $\quad$ Starshaped compact Weigarten hypersurfaces, Current topics in partial differential equations, Y.Ohya, K.Kasahara and N.Shimakura (eds), Kinokunize, Tokyo, 1985, 1-26.

[6] Cheng, Shiu Yuen; Yau, Shing Tung. On the regularity of the solution of the $n$-dimensional Minkowski problem, Comm. Pure Appl. Math. 29 (1976), no. 5, 495-516.

[7] Federer, Herbert. Curvature measures, Trans. Amer. Math. Soc. 93 (1959) 418-491.

[8] Firey, William J. Christoffel's problem for general convex bodies, Mathematika 15 1968 7-21.

[9] Guan, Pengfei; Li, YanYan. Unpublished research notes, 1995

[10] Guan, Pengfei; Li, YanYan. $C^{1,1}$ estimates for solutions of a problem of Alexandrov, Comm. Pure and Appl. Math., 50, (1997), 189-811.

[11] Guan, Pengfei; Lin, Changshou; Ma, Xinan. The Existence of Convex Body with Prescribed Curvature Measures, International Mathematics Research Notices, Vol. 2009, (2009) 19471975.

[12] Guan, Pengfei; Ma, Xinan. The Christoffel-Minkowski problem. I. Convexity of solutions of a Hessian equation, Invent. Math. 151 (2003), no. 3, 553-577. 
[13] Guan, Pengfei; Ma, Xinan. Convex solutions of fully nonlinear elliptic equations in classical differential geometry, Contemp Math. V.367, AMS. (2004), 115-128.

[14] Lewy, Hans. On differential geometry in the large. I. Minkowski's problem, Trans. Amer. Math. Soc. 43 (1938), no. 2, 258-270.

[15] Nirenberg, Louis. The Weyl and Minkowski problems in differential geometry in the large, Comm. Pure and Appl. Math., 6,(1953), pp. 337-394.

[16] Oliker, V. I. Existence and uniqueness of convex hypersurfaces with prescribed Gaussian curvatrue in spaces of constant curvature. Sem. Inst. Mate. Appl. "Giovanni Sansone", Univ. Studi Firenze, 1983.

[17] Pogorelov, A. V. Regularity of a convex surface with given Gaussian curvature, (Russian) Mat. Sbornik N.S. 31(73), (1952). 88-103.

[18] Pogorelov, A. V. Extrinsic geometry of convex surfaces, "Nauka", Moscow, 1969; English transl., Transl. Math. Mono., Vol. 35, Amer. Math. Soc., Providence, R.I., 1973.

[19] Pogorelov, A. V. The Minkowski multidimensional problem. Translated from the Russian by Vladimir Oliker. Introduction by Louis Nirenberg. Scripta Series in Mathematics. V. H. Winston \& Sons, Washington, D.C.; Halsted Press [John Wiley \& Sons], New York-TorontoLondon, 1978.

[20] Schneider, Rolf. Convex bodies: the Brunn-Minkowski theory. Encyclopedia of Mathematics and its Applications, 44. Cambridge University Press, Cambridge, 1993.

Department of Mathematics and Statistics, McGill University, Montreal, Quebec. H3A 2K6, CANADA.

E-mail address: guan@math.mcgill.ca

Department of Mathematics, University of Alabama at Birmingham, Birmingham, AL 35294

E-mail address: jfli@uab.edu

Department of Mathematics, Rutgers University, New Brunswick, NJ 08903

E-mail address: yyli@math.rutgers.edu 